\title{
On Propagation of Rayleigh Type Surface Wave in a Micropolar Piezoelectric Medium
}

\author{
Baljeet Singh1, Ritu Sindhu² \\ ${ }^{1}$ Department of Mathematics, Post Graduate Government College, Chandigarh, India \\ ${ }^{2}$ Department of Mathematics, Maharshi Dayanand University, Rohtak, India \\ Email: bsinghgc11@gmail.com
}

How to cite this paper: Singh, B. and Sindhu, R. (2016) On Propagation of Rayleigh Type Surface Wave in a Micropolar Piezoelectric Medium. Open Journal of Acoustics, 6, 3544.

http://dx.doi.org/10.4236/oja.2016.64004

Received: September 12, 2016

Accepted: December 9, 2016

Published: December 12, 2016

Copyright $\odot 2016$ by authors and Scientific Research Publishing Inc. This work is licensed under the Creative Commons Attribution International License (CC BY 4.0).

http://creativecommons.org/licenses/by/4.0/ (c) (i) Open Access

\begin{abstract}
In the present paper, the governing equations of a linear transversely isotropic micropolar piezoelectric medium are specialized for $\mathrm{x}-\mathrm{z}$ plane after using symmetry relations in constitutive coefficients. These equations are solved for the general surface wave solutions in the medium. Following radiation conditions in the half-space, the particular solutions are obtained, which satisfy the appropriate boundary conditions at the stress-free surface of the half-space. A secular equation for Rayleigh type surface wave is obtained. An iteration method is applied to compute the non-dimensional wave speed of the Rayleigh surface wave for specific material parameters. The effects of piezoelectricity, non-dimensional frequency and non-dimensional material constant, charge free surface and electrically shorted surface are shown graphically on the wave speed of Rayleigh wave.
\end{abstract}

\section{Keywords}

Piezoelectric Medium, Micro-Rotation, Transverse Isotropy, Rayleigh Wave, Wave Speed

\section{Introduction}

The materials possessing linear coupling between mechanical and electric fields are termed as piezoelectric materials. Wave propagation in piezoelectric media has numerous applications in various fields of engineering. Some problems about propagation of plane waves in piezoelectric medium are studied by Kyame [1], Pailloux [2] and Hruska [3]. Various other problems related to the phenomena of reflection and refraction of plane waves in piezoelectric materials are studied by Auld [4], Parton and Kudryavtsev [5], Galassi, et al. [6], Singh [7] and Sharma [8]. Recently Salah et al. [9] studied the propagation of Rayleigh waves in a functionally graded piezoelectric mate- 
rial half-space.

Eringen [10] [11] [12] introduced the micro-continuum field theories of solids with electro-magnetic and thermal interactions. Craciun [13] formulated the basic equations of the linear theory of piezoelectric micropolar thermoelasticity with quasi-static electric fields. Ciumasu and Vieru [14] presented the variational formulation for the free vibration of a micropolar piezoelectric body. Zhilin [15] developed a theory of the micropolar piezoelectric materials. Iesan [16] established a uniqueness result and a reciprocal theorem in the linear theory of microstretch piezoelectricity. Aouadi [17] considered the linear dynamic theory of micropolar piezoelectricity and established a reciprocity relation with two processes at different instants. Gales [18] considered the linear theory of micromorphic piezoelectricity and formulated the initial boundary value problem and presented some uniqueness results. Chen [19] derived the linear constitutive equations for micropolar electromagnetic elastic solids.

The propagation of surface waves in a transversely isotropic micropolar piezoelectric medium is not attempted so far. Following Aouadi [17], the governing equations for a transversely isotropic micropolar piezoelectric medium are formulated in $\mathrm{x}-\mathrm{z}$ plane and are solved for possible surface waves. After considering the required radiation conditions in half-space and boundary conditions at free surface, a secular equation for non-dimensional wave speed of Rayleigh surface wave is obtained. The dependence of non-dimensional wave speed on frequency, material constants and electric field is shown graphically.

\section{Governing Equations and Solution}

We consider a homogeneous and transversely isotropic micropolar piezoelectric half space. We take the origin of the coordinate system on the free surface and the positive $z$ axis along the normal into the half-space $(z \geq 0)$. We assume the components of the displacement and microrotation vectors of the form $\boldsymbol{u}=\left(u_{1}, 0, u_{3}\right)$ and $\boldsymbol{\varphi}=\left(0, \varphi_{2}, 0\right)$. Using symmetry relations in the coefficients, the governing equations given in Aouadi [17] are specialized for $\mathrm{x}-\mathrm{z}$ plane in the following from after a lengthy calculation

$$
\begin{gathered}
A_{11} \frac{\partial^{2} u_{1}}{\partial x^{2}}+\left(A_{13}+A_{56}\right) \frac{\partial^{2} u_{3}}{\partial x \partial z}+A_{55} \frac{\partial^{2} u_{1}}{\partial z^{2}}+K_{1} \frac{\partial \varphi_{2}}{\partial z}-\left(\lambda_{15}+\lambda_{31}\right) \frac{\partial^{2} \psi}{\partial x \partial z}=\rho \frac{\partial^{2} u_{1}}{\partial t^{2}} \\
A_{66} \frac{\partial^{2} u_{3}}{\partial x^{2}}+\left(A_{13}+A_{56}\right) \frac{\partial^{2} u_{1}}{\partial x \partial z}+A_{33} \frac{\partial^{2} u_{3}}{\partial z^{2}}+K_{2} \frac{\partial \varphi_{2}}{\partial x}-\lambda_{15} \frac{\partial^{2} \psi}{\partial x^{2}}-\lambda_{33} \frac{\partial^{2} \psi}{\partial z^{2}}=\rho \frac{\partial^{2} u_{3}}{\partial t^{2}} \\
B_{77} \frac{\partial^{2} \varphi_{2}}{\partial x^{2}}+B_{66} \frac{\partial^{2} \varphi_{2}}{\partial z^{2}}-\chi \varphi_{2}-K_{1} \frac{\partial u_{1}}{\partial z}-K_{2} \frac{\partial u_{3}}{\partial x}-\beta_{14} \frac{\partial^{2} \psi}{\partial x^{2}}-\beta_{36} \frac{\partial^{2} \psi}{\partial z^{2}}=\rho j \frac{\partial^{2} \varphi_{2}}{\partial t^{2}} \\
\lambda_{15} \frac{\partial^{2} u_{3}}{\partial x^{2}}+\lambda_{33} \frac{\partial^{2} u_{3}}{\partial z^{2}}+\left(\lambda_{15}+\lambda_{31}\right) \frac{\partial^{2} u_{1}}{\partial x \partial z}+\beta_{14} \frac{\partial^{2} \varphi_{2}}{\partial x^{2}}+\beta_{36} \frac{\partial^{2} \varphi_{2}}{\partial z^{2}}+\gamma_{11} \frac{\partial^{2} \psi}{\partial x^{2}}+\gamma_{33} \frac{\partial^{2} \psi}{\partial z^{2}}=0,
\end{gathered}
$$

where $A_{11}, A_{13}, A_{55}, A_{56}, A_{66}, B_{66}, \lambda_{15}, \lambda_{31}, \lambda_{33}, \beta_{14}, \beta_{36}, \gamma_{11}, \gamma_{33}$ are constitutive coefficients. $K_{1}=A_{56}-A_{55}, K_{2}=A_{66}-A_{56}, \chi=K_{2}-K_{1}$.

We seek the surface wave solution of Equations (1) to (4) in the following form 


$$
\left\{u_{1}, u_{3}, \varphi_{2}, \psi\right\}=\left\{\overline{u_{1}}(z), \overline{u_{3}}(z), \overline{\varphi_{2}}(z), \bar{\psi}(z)\right\} \mathrm{e}^{i k(x-c t)}
$$

Making use of Equation (5) in Equations (1) to (4) and applying the radiation conditions $u_{1} \rightarrow 0, u_{3} \rightarrow 0, \varphi_{2} \rightarrow 0, \psi \rightarrow 0$ as $z \rightarrow \infty$, we obtain the following particular solutions in half-space

$$
\begin{gathered}
u_{1}=\left(A_{1} \mathrm{e}^{-m_{1} z}+A_{2} \mathrm{e}^{-m_{2} z}+A_{3} \mathrm{e}^{-m_{3} z}+A_{4} \mathrm{e}^{-m_{4} z}\right) \mathrm{e}^{i k(x-c t)} \\
u_{3}=\left(\zeta_{1} A_{1} \mathrm{e}^{-m_{1} z}+\zeta_{2} A_{2} \mathrm{e}^{-m_{2} z}+\zeta_{3} A_{3} \mathrm{e}^{-m_{3} z}+\zeta_{4} A_{4} \mathrm{e}^{-m_{4} z}\right) \mathrm{e}^{i k(x-c t)} \\
\varphi_{2}=\left(\eta_{1} A_{1} \mathrm{e}^{-m_{1} z}+\eta_{2} A_{2} \mathrm{e}^{-m_{2} z}+\eta_{3} A_{3} \mathrm{e}^{-m_{3} z}+\eta_{4} A_{4} \mathrm{e}^{-m_{4} z}\right) e^{i k(x-c t)} \\
\psi=\left(\xi_{1} A_{1} \mathrm{e}^{-m_{1} z}+\xi_{2} A_{2} \mathrm{e}^{-m_{2} z}+\xi_{3} A_{3} \mathrm{e}^{-m_{3} z}+\xi_{4} A_{4} \mathrm{e}^{-m_{4} z}\right) \mathrm{e}^{i k(x-c t)}
\end{gathered}
$$

where the expressions for coupling coefficients $\xi_{i}, \zeta_{i}, \eta_{i}(i=1,2,3,4)$ and the relations between $m_{i}(i=1,2,3,4)$ are given in Appendix.

\section{Boundary Conditions}

The appropriate boundary conditions at $z=0$ are vanishing of normal and tangential force stress components, tangential couple stress component

$$
\sigma_{33}=0, \sigma_{31}=0, m_{32}=0,
$$

And vanishing of electric displacement component or electric potential

$D_{3}=0$ (for charge free case) or $\psi=0$ (for electrically shorted case), where

$$
\begin{gathered}
\sigma_{33}=A_{13} u_{1,1}+A_{33} u_{3,3}-\lambda_{35} \psi_{, 1}-\lambda_{33} \psi_{, 3}, \\
\sigma_{31}=A_{56} u_{3,1}+A_{55} u_{1,3}+\left(A_{56}-A_{55}\right) \varphi_{2}-\lambda_{31} \psi_{, 1}-\lambda_{35} \psi_{, 3}, \\
m_{32}=B_{66} \varphi_{2,3}-\beta_{36} \psi_{, 3}, \\
D_{3}=\lambda_{15} u_{1,1}+\lambda_{33} u_{3,3}+\beta_{36} \varphi_{2,3}+\gamma_{33} \psi_{, 3} .
\end{gathered}
$$

\section{Secular Equations}

The particular solutions (6) to (9) satisfy the boundary conditions (10) and (11) at the free surface $z=0$ and we obtain the following secular equation

$$
\begin{aligned}
& A_{1}^{*} B_{2}^{*} C_{3}^{*} D_{4}^{*}-A_{1}^{*} B_{2}^{*} C_{4}^{*} D_{3}^{*}-A_{1}^{*} B_{3}^{*} C_{2}^{*} D_{4}^{*}+A_{1}^{*} B_{3}^{*} C_{4}^{*} D_{2}^{*} \\
& +A_{1}^{*} B_{4}^{*} C_{2}^{*} D_{3}^{*}-A_{1}^{*} B_{4}^{*} C_{3}^{*} D_{2}^{*}-A_{2}^{*} B_{1}^{*} C_{3}^{*} D_{4}^{*}+A_{2}^{*} B_{1}^{*} C_{4}^{*} D_{3}^{*} \\
& +A_{2}^{*} B_{3}^{*} C_{1}^{*} D_{4}^{*}-A_{2}^{*} B_{3}^{*} C_{4}^{*} D_{1}^{*}-A_{2}^{*} B_{4}^{*} C_{1}^{*} D_{3}^{*}+A_{2}^{*} B_{4}^{*} C_{3}^{*} D_{1}^{*} \\
& +A_{3}^{*} B_{1}^{*} C_{2}^{*} D_{4}^{*}-A_{3}^{*} B_{1}^{*} C_{4}^{*} D_{2}^{*}-A_{3}^{*} B_{2}^{*} C_{1}^{*} D_{4}^{*}+A_{3}^{*} B_{2}^{*} C_{4}^{*} D_{1}^{*} \\
& +A_{3}^{*} B_{4}^{*} C_{1}^{*} D_{2}^{*}-A_{3}^{*} B_{4}^{*} C_{2}^{*} D_{1}^{*}-A_{4}^{*} B_{1}^{*} C_{2}^{*} D_{3}^{*}+A_{4}^{*} B_{1}^{*} C_{3}^{*} D_{2}^{*} \\
& +A_{4}^{*} B_{2}^{*} C_{1}^{*} D_{3}^{*}-A_{4}^{*} B_{2}^{*} C_{3}^{*} D_{1}^{*}-A_{4}^{*} B_{3}^{*} C_{1}^{*} D_{2}^{*}+A_{4}^{*} B_{3}^{*} C_{2}^{*} D_{1}^{*}=0
\end{aligned}
$$

where

$$
A_{i}^{*}=i k A_{13}-m_{i} \zeta_{i} A_{33}+m_{i} \xi_{i}\left(\lambda_{33}-\frac{i k \lambda_{35}}{m_{i}}\right),(i=1,2,3,4)
$$




$$
\begin{aligned}
& B_{i}^{*}=i k \zeta_{i} A_{56}-m_{i} A_{55}+\left(A_{56}-A_{55}\right) \eta_{i}-\lambda_{31} i k \xi_{i}, \\
& C_{i}^{*}=m_{i} \xi_{i} \beta_{36}-m_{i} \eta_{i} B_{66}, \\
& D_{i}^{*}=i k \lambda_{15}-m_{i} \zeta_{i} \lambda_{33}-m_{i} \eta_{i} \beta_{36}-m_{i} \xi_{i} \gamma_{33} \text { (for charge free case), } \\
& \text { Or } D_{i}^{*}=\xi_{i} \quad \text { (for electrically shorted). }
\end{aligned}
$$

\section{Particular Cases}

a) The secular Equation (12) reduces for a transversely isotropic micropolar elastic case when

$$
\lambda_{33}=0, \lambda_{35}=0, \lambda_{31}=0, \lambda_{15}=0, \beta_{36}=0, \gamma_{33}=0, \gamma_{11}=0 \text {. }
$$

b) The secular Equation (12) reduces for a transversely isotropic piezoelectric case when

$$
K_{1}=K_{2}=K=0, A_{11}=C_{11}, A_{33}=C_{33}, A_{55}=A_{66}=A_{56}=C_{44}, A_{13}=C_{13}, B_{66}=B_{77}=0 .
$$

\section{Numerical Results and Discussion}

For numerical computation of non-dimensional wave speed of Rayleigh wave, the following relevant physical constants of a transversely isotropic micropolar piezoelectric material are considered

$$
\begin{gathered}
A_{11}=17.8 \times 10^{10} \mathrm{~N} \cdot \mathrm{m}^{-2}, \quad A_{33}=18.43 \times 10^{10} \mathrm{~N} \cdot \mathrm{m}^{-2}, \quad A_{13}=7.59 \times 10^{10} \mathrm{~N} \cdot \mathrm{m}^{-2}, \\
A_{56}=1.89 \times 10^{10} \mathrm{~N} \cdot \mathrm{m}^{-2}, \quad A_{55}=4.357 \times 10^{10} \mathrm{~N} \cdot \mathrm{m}^{-2}, \quad A_{66}=4.42 \times 10^{10} \mathrm{Nm}^{-2}, \\
A_{65}=1.99 \times 10^{10} \mathrm{~N} \cdot \mathrm{m}^{-2}, \quad B_{77}=0.278 \times 10^{9} \mathrm{~N}, \quad B_{66}=0.268 \times 10^{9} \mathrm{~N}, \\
\lambda_{15}=37 \mathrm{C} \cdot \mathrm{m}^{-2}, \quad \lambda_{31}=12 \mathrm{C} \cdot \mathrm{m}^{-2}, \quad \lambda_{33}=1.33 \mathrm{C} \cdot \mathrm{m}^{-2}, \quad \lambda_{35}=0.23 \mathrm{C} \cdot \mathrm{m}^{-2} \\
\beta_{14}=0.0001 \mathrm{C}, \quad \beta_{36}=0.0002 \mathrm{C}, \quad \gamma_{11}=0.000852 \mathrm{C}^{2} \cdot \mathrm{N}^{-1} \cdot \mathrm{m}^{-2}, \\
\gamma_{33}=0.000287 \mathrm{C}^{2} \cdot \mathrm{N}^{-1} \cdot \mathrm{m}^{-2}, \quad \rho=1.74 \times 10^{3} \mathrm{~kg} \cdot \mathrm{m}^{-3}, \quad j=0.196 \mathrm{~m}^{2}
\end{gathered}
$$

For above physical constants and by using a Fortran program of Iteration method, the secular Equation (12) is solved numerically to obtain the non-dimensional speed $\sqrt{\frac{\rho c^{2}}{A_{33}}}$ for certain ranges of non-dimensional frequency and non-dimensional constant.

The variation of non-dimensional speed $\sqrt{\frac{\rho c^{2}}{A_{33}}}$ against non-dimensional frequency $\left\{\omega^{*}=\omega^{2} /\left(\frac{\chi}{\rho j}\right)\right\}$ are shown graphically in Figure 1 for charge free (CF) and electrically shorted (ES) cases. For CF case, the value of speed at $\omega^{*}=2.5$ is 1.5109 . It decreases to a value 1.4907 at $\omega^{*}=10$. This variation is shown by solid line in Figure 1 . For ES case, the value of speed at $\omega^{*}=2.5$ is 0.8036 . It decreases to value 0.5991 at $\omega^{*}=10$. This variation is shown by dotted line in Figure 1 . Comparing the solid and dotted lines in Figure 1, we can observe the effect of charge free surface over electrically 


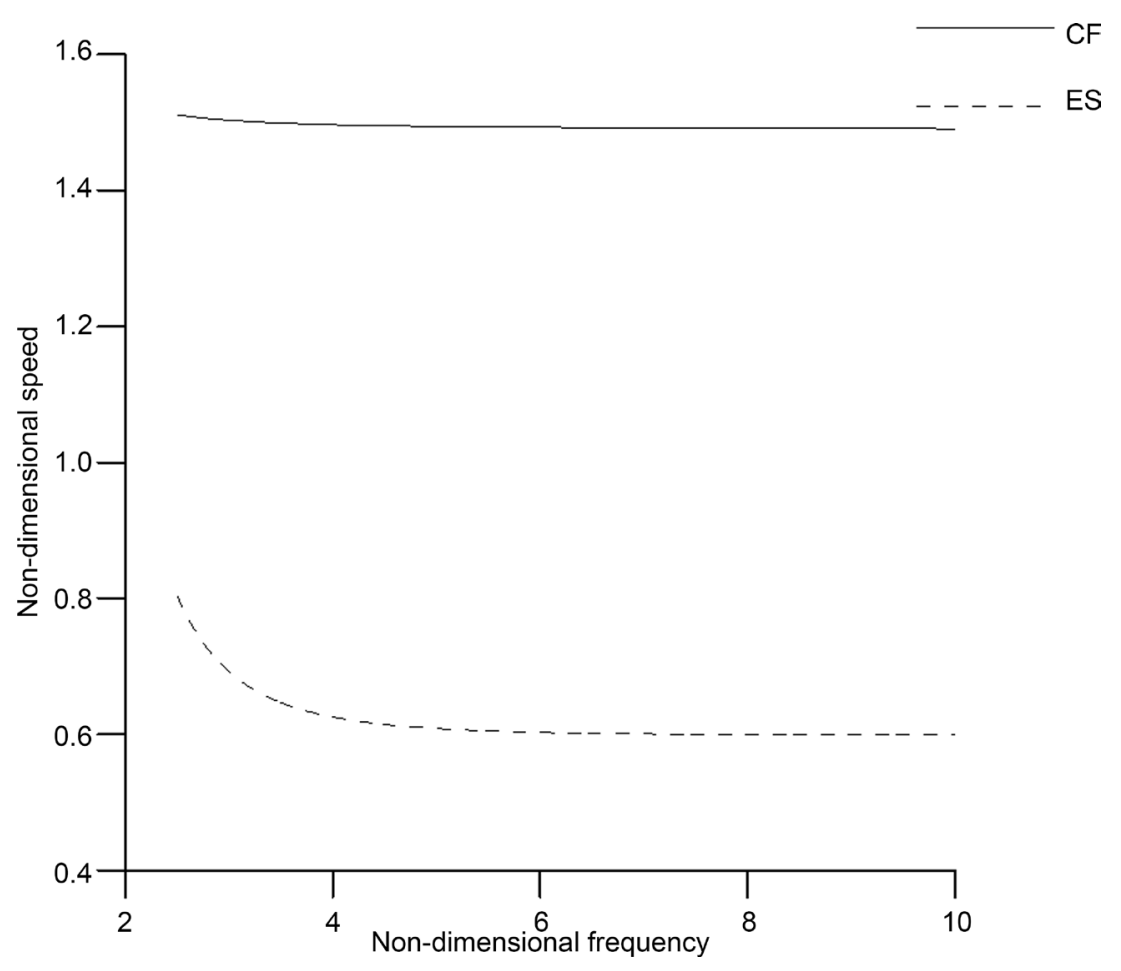

Figure 1. Variation of non-dimensional speed $\sqrt{\frac{\rho c^{2}}{A_{33}}}$ against non-dimensional frequency $\left\{\omega^{*}=\omega^{2} /\left(\frac{\chi}{\rho j}\right)\right\}$ for charge free (CF) and electrically shorted (ES) cases.

shorted surface on non-dimensional speed of the Rayleigh wave in a transversely isotropic micropolar piezoelectric solid half-space.

The variation of non-dimensional speed $\sqrt{\frac{\rho c^{2}}{A_{33}}}$ is shown graphically in Figure 2 against non-dimensional frequency $\left\{\omega^{*}=\omega^{2} /\left(\frac{\chi}{\rho j}\right)\right\}$ for charge free (CF) case to observe the piezoelectric effects. The variation non-dimensional speed as shown by solid line (transversely isotropic micropolar piezoelectric case) in Figure 2 is same as shown in Figure 1. For transversely isotropic micropolar case, the variation of non-dimensional speed is shown by dotted line in Figure 2. It has value 2.2224 at $\omega^{*}=2.5$ and it increases to value 2.8541 at $\omega^{*}=10$. The comparison of solid and dotted lines in Figure 2 shows the piezoelectric effect on non-dimensional speed of Rayleigh wave in a transversely isotropic micropolar piezoelectric solid half-space with charge free surface.

The variation of non-dimensional speed $\sqrt{\frac{\rho c^{2}}{A_{33}}}$ is shown graphically in Figure 3 against non-dimensional constant $\left(\frac{A_{11}}{A_{33}}\right)$ for charge free $(\mathrm{CF})$ case when $\omega^{*}=5,10$ 


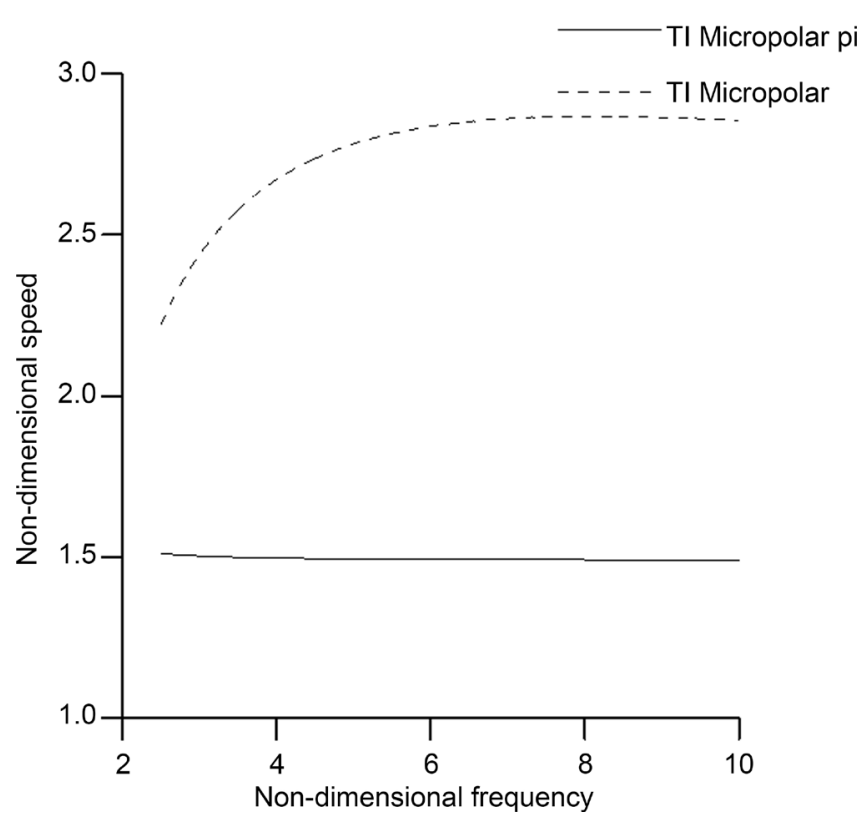

Figure 2. Piezoelectric effect on non-dimensional speed $\sqrt{\frac{\rho c^{2}}{A_{33}}}$ against non-dimensional frequency $\left\{\omega^{*}=\omega^{2} /\left(\frac{\chi}{\rho j}\right)\right\}$ for charge free (CF) case.

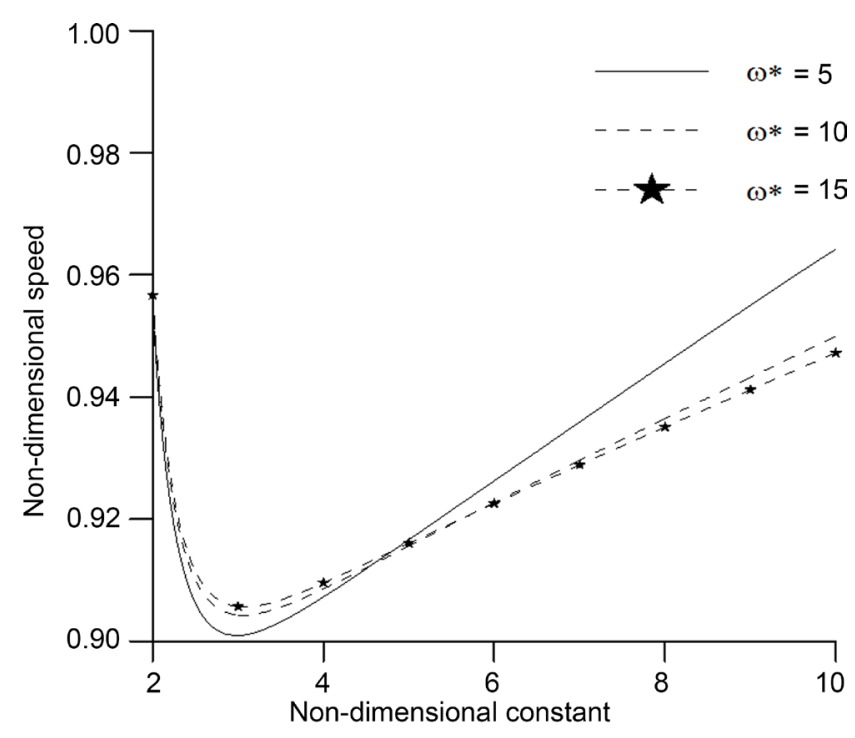

Figure 3. Variation of non-dimensional speed $\sqrt{\frac{\rho c^{2}}{A_{33}}}$ against non-dimensional constant $\left(\frac{A_{11}}{A_{33}}\right)$ for charge free (CF) case when $\omega^{*}=5,10$ and 15 .

and 15. For $\omega^{*}=5$, the non-dimensional speed is 0.9541 at $\frac{A_{11}}{A_{33}}=2$. It decreases to its minimum value 0.9010 at $\frac{A_{11}}{A_{33}}=3$ and then it increases to a maximum value 0.9642 
at $\frac{A_{11}}{A_{33}}=10$. For $\omega^{*}=10$, the non-dimensional speed is 0.9559 at $\frac{A_{11}}{A_{33}}=2$. It decreases to its minimum value 0.9043 at $\frac{A_{11}}{A_{33}}=3$ and then it increases to value 0.9499 at $\frac{A_{11}}{A_{33}}=10$. For $\omega^{*}=15$, the non-dimensional speed is 0.9567 at $\frac{A_{11}}{A_{33}}=2$. It decreases to its minimum value 0.9057 at $\frac{A_{11}}{A_{33}}=3$ and then it increases to value 0.9472 at $\frac{A_{11}}{A_{33}}=10$. The comparison of solid $\left(\omega^{*}=5\right)$, dotted $\left(\omega^{*}=10\right.$ and dotted with star $\left(\omega^{*}=15\right)$ lines in Figure 3 show the effect of non-dimensional frequency and non-dimensional material constant on non-dimensional speed of Rayleigh wave in a transversely isotropic micropolar piezoelectric solid half-space with charge free surface.

\section{Conclusion}

Using symmetry relations in constitutive coefficients and assuming the components of the displacement and microrotation vectors in the form $\boldsymbol{u}=\left(u_{1}, 0, u_{3}\right)$ and $\boldsymbol{\varphi}=\left(0, \varphi_{2}, 0\right)$, the governing equations given in Aouadi [17] are derived as a special case for transversely isotropic micropolar piezoelectric medium in $\mathrm{x}-\mathrm{z}$ plane. Rayleigh type surface wave is studied in this medium. A secular equation for non-dimensional speed of Rayleigh wave is obtained. Using Fortran program of Iteration method, the secular equation is solved numerically. The values of non-dimensional wave speed of the Rayleigh wave are obtained for a specific material modelling the medium. The nondimensional wave speed is shown graphically against the non-dimensional frequency and the non-dimensional material constant. From theory and numerical discussion, the effects of piezoelectricity, charge free surface, electrically shorted surface, non-dimensional frequency and non-dimensional material constant are observed on non-dimensional wave speed.

\section{References}

[1] Kyame, J.J. (1949) Wave Propagation in Piezoelectric Crystals. Journal of Acoustical Society of America, 21, 159-167. https://doi.org/10.1121/1.1906490

[2] Pailloux, P.M.H. (1958) Piezoelectric itecalcul des vitesses de propagation. Le Journal de Physique etle Radium, 19, 523-526. https://doi.org/10.1051/jphysrad:01958001905052300

[3] Hruska, K. (1966) The Rate of Propagation of Ultrasonic Waves in ADP and in Voigt's Theory. Czechoslovak Journal of Physics, B16, 446-453. https://doi.org/10.1007/BF01696256

[4] Auld, B.A. (1973) Acoustic Field and Waves in Solids. Wiley Interscience Publication, New York.

[5] Parton, V.Z. and Kudryavtsev B.A. (1988) Electromagnetoelasticity: Piezoelectrics and Electrically Conductive Solids. Gordan and Breach Science Publishers, New York.

[6] Galassi, C., Dinescu, M., Uchino, K. and Sayer, M. (2000) Piezoelectric Materials: Advances in Science, Technology and Applications (Nato Science Partnership Subseries 3). Springer, 
Berlin.

[7] Singh, B. (2010) Wave Propagation in a Prestressed Piezoelectric Half-Space. Acta Meccanica, 211, 337-344. https://doi.org/10.1007/s00707-009-0234-8

[8] Sharma, M.D. (2010) Piezoelectric Effect on the Velocities of Waves in an Anisotropic Piezo-Poroelastic Medium. Proceedings of the Royal Society A (Mathematical, Physical Engineering Sciences), 466, 1977-1992. https://doi.org/10.1098/rspa.2009.0534

[9] Salah, B., Njeh, A. and Ghozlen, M.H.B. (2012) A Theoretical Study of Propagation of Rayleigh Waves in Functionally Graded Piezoelectric Material (FGPM). Ultrasonics, 52, 306314. https://doi.org/10.1016/j.ultras.2011.08.016

[10] Eringen, A.C. (1999) Microcontinuum Field Theories. I. Foundations and Solids. Springer, New York. https://doi.org/10.1007/978-1-4612-0555-5

[11] Eringen, A.C. (2003) Contiinuum Theory of Micromorphic Electromagnetic Thermoelastic Solids. International Journal of Engineering Science, 41, 653-665. https://doi.org/10.1016/S0020-7225(02)00274-4

[12] Eringen, A.C. (2004) Electromagnetic Theory of Microstretch Elasticity and Bone Modelling. International Journal of Engineering Science, 42, 231-242. https://doi.org/10.1016/S0020-7225(03)00288-X

[13] Craciun, I.A. (1995) Uniqueness Theorem in the Linear Theory of Piezoelectric Micropolar Thermoelasticity. International Journal of Engineering Science, 33, 1027-1036. https://doi.org/10.1016/0020-7225(94)00106-T

[14] Ciumasu, S.G. and Vieru, D. (1999) Variational Formulations for the Vibration of a Micropolar Piezoelectric Body. Journal of Acoustical Society of America, 105, 1240-1245. https://doi.org/10.1121/1.425960

[15] Zhilin, P.A. and Kolpakov, Y.E. (2005) A Micro-Polar Theory for Piezoelectric Materials, Lecture at XXXIII Summer School-Conference "Advanced Problems in Mechanics", St. Petersburg.

[16] Iesan, D. (2006) On the Microstretch Piezoelectricity. International Journal of Engineering Science, 44, 819-829. https://doi.org/10.1016/j.ijengsci.2006.05.007

[17] Aouadi, M. (2008) Aspects of Uniqueness in Micropolar Piezoelectric Bodies. Mathematics and Mechanics of Solids, 13, 499-512. https://doi.org/10.1177/1081286507077106

[18] Gales, C. (2012) Some Results in Micromorphic Piezoelectricity. European Journal of Mechanics-A/Solids, 31, 37-46. https://doi.org/10.1016/j.euromechsol.2011.06.014

[19] Chen, J. (2013) Micropolar Theory of Flexoelectricity. Journal of Advanced Mathematics and Applications, 1, 1-6. 


\section{Nomenclature}

$\boldsymbol{u}:$ the displacement vector.

$\varphi$ : the microrotation vector.

$\rho:$ the mass density.

$j$ : the micro-inertia.

$\psi$ : the electrostatic potential.

$k$ : the wave number.

$c:$ the phase velocity of the wave.

$\omega=k c$ : the angular frequency.

$\sigma_{i j}:$ the force stress tensor.

$m_{i j}$ : the couple stress tensor.

$\xi_{i}, \zeta_{i}, \eta_{i}(i=1,2,3,4)$ : the coupling coefficients.

\section{Appendix}

The relations between $m_{i},(i=1,2,3,4)$ are given as

$$
\begin{aligned}
& m_{1}^{2}+m_{2}^{2}+m_{3}^{2}+m_{4}^{2}=S_{1}, \\
& m_{1}^{2} m_{2}^{2}+m_{2}^{2} m_{3}^{2}+m_{3}^{2} m_{4}^{2}+m_{4}^{2} m_{1}^{2}=S_{2} \text {, } \\
& m_{1}^{2} m_{2}^{2} m_{3}^{2}+m_{2}^{2} m_{3}^{2} m_{4}^{2}+m_{3}^{2} m_{4}^{2} m_{1}^{2}=S_{3} \text {, } \\
& m_{1}^{2} m_{2}^{2} m_{3}^{2} m_{4}^{2}=S_{4} \text {, } \\
& S_{1}=\left[k ^ { 2 } \left(A_{33} A_{55} \gamma_{33} P+A_{33} A_{55} B_{66} \gamma_{11}+A_{55} P \lambda_{33}^{2}+A_{55} B_{66} \gamma_{33} N+A_{55} N \beta_{36}^{2}+L A_{33} B_{66} \gamma_{33}+L A_{33} \beta_{36}^{2}\right.\right. \\
& \left.+L B_{66} \lambda_{33}^{2}-B_{66} \gamma_{33} M^{2}-\beta_{36}^{2} M^{2}+A_{33} B_{66} R^{2}+2 A_{33} A_{55} \beta_{14} \beta_{36}+2 A_{55} B_{66} \lambda_{33} \lambda_{15}-2 M B_{66} \lambda_{33} R\right) \\
& \left.-K_{1}^{2} A_{33} \gamma_{33}-K_{1}^{2} \lambda_{33}^{2}\right] /\left(A_{33} A_{55} B_{66} \gamma_{33}+A_{33} A_{55} \beta_{36}^{2}+A_{55} B_{66} \lambda_{33}^{2}\right) \text {, } \\
& S_{2}=\left[k ^ { 4 } \left(A_{55} B_{66} \gamma_{11} N+A_{33} A_{55} \gamma_{11} P+A_{33} A_{55} \beta_{14}^{2}+A_{55} \gamma_{33} N P+A_{33} \gamma_{33} L P+B_{66} \gamma_{33} L N+L N \beta_{36}^{2}+A_{55} B_{66} \lambda_{15}^{2}\right.\right. \\
& +\lambda_{33}^{2} L P+A_{33} B_{66} \gamma_{11} L+A_{33} P R^{2}+B_{66} N R^{2}-B_{66} \gamma_{11} M^{2}-\gamma_{33} P M^{2}+2 A_{33} \beta_{14} \beta_{36} L+2 A_{55} \beta_{14} \beta_{36} N \\
& \left.+2 A_{55} \lambda_{33} \lambda_{15} P+2 B_{66} \lambda_{33} \lambda_{15} L-2 \beta_{14} \beta_{36} M^{2}-2 B_{66} \lambda_{15} M R-2 \lambda_{33} M P R\right)-k^{2}\left(A_{33} \gamma_{11} K_{1}^{2}+A_{55} \gamma_{33} K_{2}^{2}\right. \\
& \left.\left.+\gamma_{33} N K_{1}^{2}+2 \lambda_{33} \lambda_{15} K_{1}^{2}-2 \gamma_{33} M K_{1} K_{2}-2 \lambda_{33} R K_{1} K_{2}\right)\right] /\left(A_{33} A_{55} B_{66} \gamma_{33}+A_{33} A_{55} \beta_{36}^{2}+A_{55} B_{66} \lambda_{33}^{2}\right), \\
& S_{3}=\left[k ^ { 6 } \left(A_{55} \gamma_{11} N P+A_{55} \beta_{14}^{2} N+B_{66} \gamma_{11} L N+A_{33} \gamma_{11} L P+A_{33} \beta_{14}^{2} L+\gamma_{33} L N P+A_{55} \lambda_{15}^{2} P+B_{66} \lambda_{15}^{2} L\right.\right. \\
& \left.-\gamma_{11} M^{2} P-\beta_{14}^{2} M^{2}+P R^{2} N+2 \beta_{14} \beta_{36} L N+2 \lambda_{33} \lambda_{15} L P-2 \lambda_{15} M P R\right)-k^{4}\left(A_{55} \gamma_{11} K_{2}^{2}+\gamma_{33} L K_{2}^{2}\right. \\
& \left.\left.+\gamma_{11} N K_{1}^{2}+\lambda_{15}^{2} K_{1}^{2}+K_{2}^{2} R^{2}-2 K_{1} K_{2} \gamma_{11} M-2 K_{1} K_{215} R\right)\right] /\left(A_{33} A_{55} B_{66} \gamma_{33}+A_{33} A_{55} \beta_{36}^{2}+A_{55} B_{66} \lambda_{33}^{2}\right) \\
& S_{4}=\left[k^{8}\left(\gamma_{11} L N P+\beta_{14}^{2} L N+\lambda_{15}^{2} L P\right)-k^{6}\left(\gamma_{11} L K_{2}^{2}\right)\right] /\left(A_{33} A_{55} B_{66} \gamma_{33}+A_{33} A_{55} \beta_{36}^{2}+A_{55} B_{66} \lambda_{33}^{2}\right) \\
& L=\left(A_{11}-\rho c^{2}\right), M=\left(A_{13}+A_{56}\right), N=\left(A_{66}-\rho c^{2}\right), \quad, \\
& P=\left(B_{77}-\rho j c^{2}+\frac{\chi}{k^{2}}\right), R=\left(\lambda_{15}+\lambda_{31}\right)
\end{aligned}
$$

and 


$$
\begin{gathered}
\zeta_{i}=\frac{\left[p_{i} q_{i}+\left(u_{i}-v_{i} r_{i}\right)\right]}{q_{i} s_{i}+t_{i} r_{i}}, \xi_{i}=\frac{p_{i}-s_{i} \zeta_{i}}{r_{i}}, \frac{\eta_{i}}{k}=\frac{\left[w_{i}-\left(i M \zeta_{i} \frac{m_{i}}{k}-i R \xi_{i} \frac{m_{i}}{k}\right)\right]}{K_{1} \frac{m_{i}}{k}} \\
p_{i}=\left(i K_{2} A_{55} \frac{m_{i}^{2}}{k^{2}}-i K_{2} L-i M K_{1} \frac{m_{i}^{2}}{k^{2}}\right), \\
q_{i}=\left\{\left(\beta_{36} \frac{m_{i}^{2}}{k^{2}}-\beta_{14}\right)^{2}+\left(\gamma_{33} \frac{m_{i}^{2}}{k^{2}}-\gamma_{11}\right)\left(B_{66} \frac{m_{i}^{2}}{k^{2}}-P\right)\right\}, \\
r_{i}=\left(R K_{2} \frac{m_{i}}{k}-\lambda_{15} K_{1} \frac{m_{i}}{k}+\lambda_{33} K_{1} \frac{m_{i}^{3}}{k^{3}}\right), \\
s_{i}=\left(N K_{1} \frac{m_{i}}{k}-M K_{2} \frac{m_{i}}{k}-A_{33} K_{1} \frac{m_{i}^{3}}{k^{3}}\right), \\
t_{i}=-\frac{m_{i}}{k} K_{1}\left(\beta_{36} \frac{m_{i}^{2}}{k^{2}}-\beta_{14}\right), v_{i}=i R \frac{m_{i}}{k}\left(B_{66} \frac{m_{i}^{2}}{k^{2}}-P\right), w_{i}=\left(A_{55} \frac{m_{i}^{2}}{k^{2}}-L\right) .
\end{gathered}
$$

Submit or recommend next manuscript to SCIRP and we will provide best service for you:

Accepting pre-submission inquiries through Email, Facebook, LinkedIn, Twitter, etc. A wide selection of journals (inclusive of 9 subjects, more than 200 journals)

Providing 24-hour high-quality service

User-friendly online submission system

Fair and swift peer-review system

Efficient typesetting and proofreading procedure

Display of the result of downloads and visits, as well as the number of cited articles

Maximum dissemination of your research work

Submit your manuscript at: http://papersubmission.scirp.org/

Or contact oja@scirp.org 